\title{
THE DISTANCE, ABSOLUTE MAGNITUDE AND SPACE MOTION OF ALPHA ORIONIS
}

\author{
R. F. WING ${ }^{1}$, E. F. GUINAN ${ }^{2}$ \\ 1 Ohio State University, Columbus OH, USA \\ 2 Villanova University, Villanova PA, USA
}

Alpha Orionis (Betelgeuse) is the brightest, and presumably the nearest, of the M-type supergiants. It is also one of the least reddened. These features make it important for the calibration of the absolute magnitudes and intrinsic colors of $M$ supergiants. However, its distance has been difficult to establish, and there has been considerable debate concerning its reddening.

The Hipparcos parallax for $\alpha$ Ori is $7.63 \pm 1.64$ mas, corresponding to a distance of $131 \pm 30 \mathrm{pc}$ and a true distance modulus $(m-M)_{0}$ of $5.59 \pm 0.48 \mathrm{mag}$. If we adopt $\langle V\rangle=0.50$ and $E(B-V)$ $=0.05$, the mean absolute visual magnitude becomes $\left\langle M_{V}\right\rangle=-5.24$, a result consistent with a luminosity classification of Iab.

The CN strength of $\alpha$ Ori measured in the near infrared also indicates luminosity class Iab. Furthermore, the star's absolute magnitude at $1.04 \mu \mathrm{m}$, for the Hipparcos distance, is -8.34 , in agreement with the M2 Iab supergiants in $h \& \chi$ Per for a distance modulus of $(m-M)_{\circ}=12.0$.

From the Hipparcos proper-motion components and distance and a published radial velocity, we derive a space motion of $27.8 \mathrm{~km} \mathrm{~s}^{-1}$ with components $\mathrm{U}=+19.7, \mathrm{~V}=-7.3$, and $\mathrm{W}=-1.5 \mathrm{~km} \mathrm{~s}^{-1}$ with respect to the Sun. This motion is not very different from the local standard of rest. It seems unlikely that $\alpha$ Ori, at a distance only one-third that of the Trapezium, is physically associated with the Orion Complex.

At $131 \mathrm{pc}$, the observed optical angular diameter of $\alpha$ Ori, 0.055 arcsec, translates to a linear diameter of 7.2 AU. Centered on the Sun, its photosphere would be in the asteroid belt. Its angular diameter measured by the Hubble Space Telescope in the UV (corresponding to the size of the chromosphere) is a factor of 2 larger. Surface features detected interferometrically are believed to be $\sim 10 \%$ of the diameter in extent, corresponding to $0.7 \mathrm{AU}$ or $10^{8} \mathrm{~km}$.

\section{ON THE MASS-LUMINOSITY RELATION}

\author{
P. LAMPENS ${ }^{1}$, J. KOVALEVSKY ${ }^{2}$, M. FROESCHLE' ${ }^{2}$, G. RUYMAEKERS ${ }^{1}$ \\ ${ }^{1}$ Royal Observatory of Belgium, Ringlaan 3, B-1180 Brussels, Belgium \\ 2 Observatoire de la Côte d'Azur, CERGA, UMR CNRS 6527, 06130 Grasse, France
}

The new Hipparcos parallaxes and photometry are used to determine individual masses and absolute (bolometric) magnitudes for the components of nearby visual binaries with good to very good orbits. The impact on the mass-luminosity relation (MLR) in the range $0<M_{B o l}<+7.5$ mag is then evaluated.

We selected 335 visual binaries within $50 \mathrm{pc}\left(\sigma_{\pi} / \pi<10 \%\right)$ for a full error analysis of their orbits by computing the covariance matrix of the orbital elements with Pourbaix' (1994) algorithm. Using $\Delta H_{p}$ and $\pi$, we estimated fractional and component masses as well as absolute magnitudes with their respective errors: 52 binary systems have relative mass errors smaller than $15 \%$. Lutz and Kelker (1973) corrections have been applied to both data types. A new relation $B C\left(H_{p}\right)$ as a function of $T_{\text {eff }}$ was obtained for the conversion to bolometric magnitudes. A doubly weighted linear regression model was applied next (Babu and Feigelson, 1996): we derived a "mean" slope $3.82 \pm 0.07$ and zero point $4.94 \pm 0.03$ for the MLR, assuming a linear relationship.

Conclusions: a) the improvement of the data on masses by Hipparcos is largely quantitative; b) not all systems agree: small fluctuations from a "mean" MLR are found as expected from evolutionary or abundance effects; c) the break in the slope of the MLR near $M_{B o l}=+7$ cannot. be assessed due to a lack of low-mass binaries in our sample.

\section{References}

Babu G.J., Feigelson E.D. (1996), "Astrostatistics", ed. Chapmann and Hall, p. 127.

Lutz T.E., Kelker D.H. (1973), PASP 85, 573

Pourbaix D. (1994), $A \& A 290,6$. 